\title{
Two Simple Approaches to Sol-Gel Transition
}

\author{
Alexander I. Olemskoi ${ }^{1}$ \\ Sumy State University \\ Rimskii-Korsakov St. 2, Sumy, 40007 Ukraine \\ Ivan Krakovský \\ Department of Macromolecular Physics, Faculty of Mathematics \& Physics \\ Charles University \\ V Holešovičkách 2, 18000 Prague 8, Czech Republic
}

\begin{abstract}
We represent a theory of polymer gelation as an analogue of liquid-glass transition in which elastic fields of stress and strain shear components appear spontaneously as a consequence of the cross-linking of macromolecules. This circumstance is explained on the basis of obvious combinatoric arguments as well as a synergetic Lorenz system, where the strain acts as an order parameter, a conjugate field is reduced to the elastic stress, and the number of cross-links is a control parameter. Both the combinatoric and synergetic approaches show that an anomalous slow dependence of the shear modulus on the number of cross-links is obtained.
\end{abstract}

PACS: 05.70Ln, $47.17+\mathrm{e}, 61.43 \mathrm{Fs}$

Keywords: Polymer gelation; sol-gel transition; Shear stress/strain; Shear modulus

\section{INTRODUCTION}

Within the phenomenological framework the basic distinction between a liquid and a glass consists in character of the relaxation law of shear components of the elastic stress: if in an ideal glass they are kept infinitely long, in a liquid

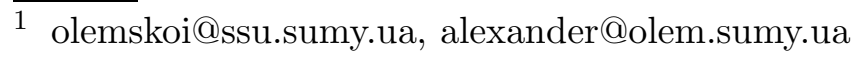


such relaxation proceeds in the finite time $\tau=\eta / G$, where $\eta$ is the dynamical shear viscosity and $G$ is the shear modulus [1]. In naive manner it is possible to assume, that a glass transition is caused purely by kinetic effect of a liquid freezing, for which viscosity $\eta$ gets infinite value for a finite value of the shear modulus $G$ [2]. However in the course of usual second-order phase transition, where infinite increase of the $\tau$ at critical point is also observed the situation is reverse. Really, proceeding from the viscoelastic liquid to a general case, one has $\tau=\chi / \gamma$, where $\chi$ is the generalized susceptibility and $\gamma$ is the kinetic coefficient (in the case under consideration they are reduced to the quantities $G^{-1}, \eta^{-1}$, respectively) [3]. The infinite increase of a susceptibility $\chi$ occurs and a kinetic coefficient $\gamma$ does not manifest any peculiarity at the phase transition. In our case, this is equivalent to the situation when the shear modulus $G$ is approaching zero value at a fixed viscosity $\eta$. This situation corresponds to a viscoelastic transition [4].

Usually under a glass transition, the transition into a glassy state which occurs during fast cooling of a liquid is understood. However, an analogous type of transition occurs during polymer network formation (polymer gelation) from monomers or linear macromolecules ( $s o l$ ) by means of a chemical reaction at constant temperature. This transition is referred to as the sol-gel transition. In such a case, thermodynamic peculiarities are observed as, e.g., appearance of the shear modulus when the system reaches a critical point. ${ }^{2}$ The first theoretical representations of such type transitions have been elaborated in classical works by Flory [5] - [7]. Modern description of the glassy state of the macromolecule networks (see [8] - [10]) is based on pioneering contributions to the theory of soft condensed matter: the Deam-Edwards theory [11] of a cross-linked tangled macromolecule, and the Edwards-Anderson theory [12] of spin glass.

The aim of this paper is to elaborate the simplest theoretical scheme so that the transition occurring during polymer gelation can be explained on the base of obvious combinatoric arguments (Section 2) as well as within framework of a synergetic theory (Section 3). In the former case, the critical point and behaviour of the system behind the critical point are obtained using information about the cross-linking properties of the constituent parts of the system (monomers) and extent of the cross-linking process, only. In Section 3, the starting point is that a sol-gel transition is ensured by self-organization of the elastic fields of stress and strain shear components and the number of crosslinks. Both the combinatoric approach and the synergetic one allow to obtain anomalous dependence of the shear modulus on the number of cross-links.

2 Of course, when the temperature of the polymer network formed is decreased the kinetic glass transition in above sense occurs, as well. 


\section{Combinatoric approach}

The gelation process has been described first by Flory[5] and Stockmayer[13]. They used a laborious approach based on combinatoric considerations of the most probable composition of the system. A much more effective variant of this approach, exploiting theory of branching processes, has been developed later by Dobson and Gordon [14,15]. This approach allows also an analysis of structural details of the system behind the critical point, see, e.g. [16].

As the simplest case let us consider the gelation process in the system which at the beginning consists of a large number of monomers $\mathcal{N}$, wearing $f$ functional terminal groups of independent reactivity. Monomers react mutually and irreversibly via their terminal groups and if $f>2$, branched molecules of increasing size and complexity are formed progressively in the system. Extent of the reaction is described by the conversion of terminal groups $\alpha$, which is defined as the ratio of the number of groups consumed by the reaction at given time and the starting number of groups. Eventually, when a critical conversion $\alpha_{\mathrm{c}}$ is achieved a molecule of macroscopic dimensions ( $\mathrm{gel}$ ) appears in the system [5], [6]. Gel contains cycles of long sequences of linked monomers and, consequently, attains elastic properties. Flory [7] has shown that shear modulus of gel $G$ is proportional to its "cycle rank" $\xi$ defined as the number of "superfluous" links formed in gel which can be cut without breaking integrity of gel. In other words, cycle rank is the number of cuts needed for elimination of all cycles from gel.

The key role in the approach is played by the extinction probability $v$, which is a probability that a link formed in the process has just a finite continuation. It can be shown easily [14] that for the system considered here the extinction probability can be obtained as a root of the equation

$$
v=(1-\alpha+\alpha v)^{f-1}
$$

satisfying the condition $0 \leq v \leq 1$. Eq.(1) expresses fact that a given link has a finite continuation in a direction only if $f-1$ functional groups remaining on the monomer connected by the link are either unreacted (with probability $1-$ $\alpha$ ) or reacted giving rise to links with finite continuation only (with probability $\alpha v$ ). Below the critical conversion, the only solution of Eq.(1) is $v=1$, i.e., only molecules of finite size are formed in the system. However, behind the critical conversion monomers can be found either in sol or in gel: $v<1$. As a measure of the "distance" from the critical conversion let us introduce the parameter $\epsilon$ defined as

$$
\epsilon \equiv \alpha-\alpha_{\mathrm{c}}
$$


and expand the extinction probability in a series:

$$
v=1+A_{1} \epsilon+A_{2} \epsilon^{2}+\ldots
$$

Substituting Eqs.(2) and (3) into Eq.(1), expressions for the critical conversion and the parameters $A_{i}$ are obtained as

$$
\begin{aligned}
& \alpha_{\mathrm{c}}=\frac{1}{f-1}, \\
& A_{1}=-2 \frac{(f-1)^{2}}{f-2}, \\
& A_{2}=\frac{4}{3} \frac{(2 f-3)(f-1)^{3}}{(f-2)^{2}} .
\end{aligned}
$$

The expression for the cycle rank can be found in the following way. Obviously, to join $\mathcal{N}$ monomers into a cycle-free structure, $\mathcal{N}-1$ links are necessary. By definition, the cycle rank $\xi$ is the number of "superfluous" links formed in gel, i.e., the difference between the number $\mathrm{N}_{\mathrm{G}}$ of all links formed in gel and the number $\mathcal{N}_{\mathrm{G}}-1$ of links sufficient to join together $\mathcal{N}_{\mathrm{G}}$ monomers of gel:

$$
\xi \equiv \mathrm{N}_{\mathrm{G}}-\left(\mathcal{N}_{\mathrm{G}}-1\right) \simeq \mathrm{N}_{\mathrm{G}}-\mathcal{N}_{\mathrm{G}}
$$

as $\mathrm{N}_{\mathrm{G}}, \mathcal{N} \gg 1$. Correspondingly, the number of links formed in gel is the difference between the numbers of links formed in the total system and in sol, i.e.,

$$
\mathrm{N}_{\mathrm{G}}=\frac{1}{2} \mathcal{N} \alpha f\left(1-v^{2}\right)
$$

as any link in sol has to have finite continuations in two directions. On the other hand, the number $\mathcal{N}_{\mathrm{G}}$ of monomers incorporated in gel is the difference between the total number of monomers and the number of monomers in sol which is made of monomers with links of only finite continuation:

$$
\mathcal{N}_{\mathrm{G}}=\mathcal{N}-\mathcal{N}(1-\alpha+\alpha v)^{f}
$$

By virtue of Eqs.(7) - (9), one gets for cycle rank of the system considered

$$
\frac{\xi}{\mathcal{N}}=(1-\alpha+\alpha v)^{f}+\frac{1}{2} \alpha f\left(1-v^{2}\right)-1
$$


Finally, substituting Eqs.(3),(5) and (6) into the formula (10), one gets the necessary expansion:

$$
\frac{\xi}{\mathcal{N}}=\frac{2}{3} \frac{(f-1)^{4}}{(f-2)^{2}} f \epsilon^{3}+O\left(\epsilon^{4}\right) .
$$

Respectively, the weight fraction of gel $w_{\mathrm{G}} \equiv \mathcal{N}_{\mathrm{G}} / \mathcal{N}$ is determined by Eq.(9) to read

$$
w_{\mathrm{G}}=2 \frac{(f-1)^{2}}{f-2} \epsilon+O\left(\epsilon^{2}\right)
$$

So, if the critical exponent of the gel weight fraction is equal 1 as usual, this for the cycle rank is anomalous large being equal 3. It is worthwhile to note that combinatoric approach is based exclusively on the information about the functionality of the monomers and extent of the chemical reaction between the monomers in the system considered.

\section{Synergetic approach}

Now, let us consider the polymer network as a viscoelastic continuum matter that is characterized by the shear modulus $G$ and the shear viscosity $\eta$. Process of polymer gelation is determined by the number of the cross-links $N$, which value is different from a stationary magnitude $N_{0}$ at a time $t$. Therein, an elastic state of the polymer is defined by the shear component of the proper (internal) values of deformation $\varepsilon(t)$ and stress $\sigma(t)$. The keypoint is that these values are not reduced to the external elastic deformation $e \ll 1$ and stress $\sigma_{e} \ll G$, in particular they can get large magnitudes $\varepsilon \sim 1, \sigma \sim G$.

Our consideration of evolution of the elastic continuum with the internal structure is stated on the phenomenological equations by Maxwell-Kelvin [1]

$$
\begin{gathered}
\frac{d \varepsilon}{d t}=-\frac{\varepsilon}{\tau}+\frac{\sigma}{\eta}, \\
\frac{d \sigma}{d t}=-\frac{\sigma}{\tau_{\sigma}}+g_{\sigma} \varepsilon N .
\end{gathered}
$$

Here we introduce a macroscopic relaxation time $\tau$ for the strain and a microscopic one $\tau_{\sigma}$ for the stress, as well as a constant $g_{\sigma}>0$ of the positive feedback between the deformation $\varepsilon$ and the number of cross-links $N$. Within 
the microscopic interval $t \gg \tau_{\sigma}$, steady-state condition $d \sigma / d t=0$ in Eq.(14) leads to the Hooke law with the microscopic shear modulus

$$
G_{\sigma} \equiv \tau_{\sigma} g_{\sigma} N
$$

being determined by the number of cross-links $N$. Respectively, within a macroscopic interval $t \gg \tau$ Eq.(13) gives the magnitude $G \equiv \eta / \tau$ that is characteristic for the usual modulus of the viscoelastic matter. Lastly, a variation rate $d N / d t$ of the internal degree of freedom is supposed to be determined by the equation

$$
\frac{d N}{d t}=\frac{N_{0}-N}{\tau_{N}}-g_{N} \sigma \varepsilon
$$

where $\tau_{N}$ is a mesoscopic relaxation time, $g_{N}>0$ is constant of negative feedback between the deformation $\varepsilon$ and the stress $\sigma$. Within a mesoscopic interval $\tau_{N} \ll t \ll \tau$, Eq.(16) determines a steady-state value

$$
N=N_{0}-\tau_{N} g_{N} \sigma \varepsilon
$$

that is smaller than the magnitude $N_{0}$ fixed by external conditions due to the fact that the elastic energy is proportional to the product $\sigma \varepsilon$.

System of Eqs.(13), (14) and (16) is known in synergetics [17] as the Lorenz system where the deformation $\varepsilon$, the stress $\sigma$ and the number of cross-links $N$ play roles of an order parameter, a conjugate field and a control parameter, respectively. It is very important for following considerations that the relation between micro-, meso- and macroscopic values of the relaxation times

$$
\tau_{\sigma}, \tau_{N} \ll \tau
$$

is satisfied. Due to this condition the evolution of the quantities $\sigma, N$ turns out to be subordinated to the long-time variation of $\varepsilon$. A peculiarity of the Lorenz system consists in linear character of the equation (13) for the order parameter $\varepsilon$ and in non-linearity of equations (14), (16) for the conjugate field $\sigma$ and the control parameter $N$. The negative nature of non-linearity in Eq.(16) means a decrease of the number $N$ of cross-links. Evidently, this fact reflects Le Chatelier principle. A non-linear term in Eq.(14) for a field $\sigma$ describes the positive feedback causing the system self-organization.

Expressions (13), (14) and (16) form the complete system of equations determining the polymer cross-linking behaviour. Because of a slow evolution, the order parameter $\varepsilon(t)$ subordinates variations of quantities $\sigma(t), N(t)$, so 
that one can take $d \sigma / d t=d N / d t=0$ within the framework of the adiabatic approximation [17]. Then $N, \sigma$ are expressed in terms of $\varepsilon$ by the equations:

$$
\begin{aligned}
& N=\frac{N_{0}}{1+\varepsilon^{2} / \varepsilon_{m}^{2}}, \quad \varepsilon_{m}^{-2} \equiv \tau_{\sigma} \tau_{N} g_{\sigma} g_{N} ; \\
& \sigma=G_{0} \frac{\varepsilon}{1+\varepsilon^{2} / \varepsilon_{m}^{2}}, \quad G_{0} \equiv \tau_{\sigma} g_{\sigma} N_{0} .
\end{aligned}
$$

In accordance with Eq.(19) the number of cross-links $N$ decreases monotoneously with increase of the strain $\varepsilon$ from the value $N_{0}$ at $\varepsilon=0$ to $N_{0} / 2$ at $\varepsilon=\varepsilon_{m} \cdot{ }^{3}$ In Eq.(20) the elastic stress in terms of the strain has the linear form of the Hooke law at $\varepsilon \ll \varepsilon_{m}$ with the effective shear modulus $G_{0}$. Then, at $\varepsilon=\varepsilon_{m}$ the function $\sigma(\varepsilon)$ has a maximum and at $\varepsilon>\varepsilon_{m}$ it decreases which is physically meaningless. Thus, the constant $\varepsilon_{m}$, defined by the second equation (19), has the meaning of the maximum achievable strain.

Substituting Eq.(20) in Eq.(13) we find the equation describing evolution of a system in the course of the sol-gel transition:

$$
\frac{d \varepsilon}{d t}=-\gamma \frac{\partial E}{\partial \varepsilon}, \quad \gamma \equiv \frac{\varepsilon_{m}^{2}}{\tau T N_{0}}
$$

where constant $\gamma$ plays a role of the kinetic coefficient. Behaviour of the system under consideration is determined by the dependence $E(\varepsilon)$ of the elastic energy on the strain:

$$
E \equiv \frac{T N_{0}}{2}\left[\frac{\varepsilon^{2}}{\varepsilon_{m}^{2}}-\frac{N_{0}}{N_{c}} \ln \left(1+\frac{\varepsilon^{2}}{\varepsilon_{m}^{2}}\right)\right]
$$

where the characteristic value of the number of cross-links is introduced

$$
N_{c} \equiv \frac{\eta}{\tau_{\sigma} g_{\sigma}}
$$

At $N_{0} \leq N_{c}$ dependence (22) is monotoneously increasing with a minimum at the point $\varepsilon=0$. It means that in the stationary state $(\dot{\varepsilon}=0)$ the elastic strain

3 Obviously this decrease is caused by the negative feedback in Eq.(16), that is reflection of Le Chatelier principle for analyzes problem. Actually, a liquid selforganization, resulting in a sol-gel transition, is caused by the positive feedback between the strain $\varepsilon$ and the number of cross-links $N$ in Eq.(14). Hence, the increase of the number of cross-links should intensify the self-organization effect. However, according to Eq.(19) system behaves in such way that the consequence of selforganization, i.e., growth of the elastic strain, leads to decrease of its origin - the number of cross-links. 
is not spontaneous. Thus, a liquid state is realized, in which the strain caused by the external stress relaxes during the time

$$
\tau_{e f}=\tau\left(1-N_{0} / N_{c}\right)^{-1}
$$

The relaxation time increases infinitely when the number of cross-links $N_{0}$ reaches the critical value $N_{c}$ and at $N_{0}>N_{c}$ the system undergoes a sol-gel transition. In the gel state the multiplier $1 / 2$ appears in the dependence (24), and the minimum of the elastic energy corresponds to the elastic strain

$$
\frac{\varepsilon^{2}}{\varepsilon_{m}^{2}}=\frac{N_{c}}{N_{0}} \frac{N_{0}-N_{c}}{N_{c}} \equiv \frac{\epsilon}{1+\epsilon}
$$

where we introduce the distance from the critical value $N_{c}$

$$
\epsilon \equiv \frac{N_{0}-N_{c}}{N_{c}}
$$

being equivalent to the definition (2). Inserting Eq.(24) into the dependence (22), we obtain the elastic energy of the steady-state:

$$
E_{0} \equiv E\left(\varepsilon_{0}\right)=-\frac{T N_{0}}{4} \epsilon^{2}+O\left(\epsilon^{3}\right)
$$

As would be expected, this energy is proportional to the second power of the parameter (26) and is negative in nature (the latter means the energy benefit of the gel state in comparison with the liquid state).

Taking into account that the glassy state is determined by density of the localized monomers, let us find now the shear modulus of the appeared gel state. It is principally important in our considerations that the gel state is determined by the value of the Deam-Edwards parameter of localization $\omega$ [11] which is supposed to be proportional to the square of the proper strain $\varepsilon_{0}$ of the matter. Under the condition of the appearance of the elastic strains $e$, a generalized Deam-Edwards parameter $\omega(e)$ has to be considered which is related to the condition $\omega(e=0) \equiv \omega$. Then, expanding the function $\omega(e)$ into a series and keeping the first two terms only, one obtains

$$
\omega(e) \simeq \omega\left(1+e^{2}\right), \quad \omega \propto \varepsilon_{0}^{2}, \quad e \ll 1
$$

By virtue of the parity condition $\omega(e)=\omega(-e)$, this expansion does not contain a linear term. 4 Because of that the total strain $\varepsilon \propto \sqrt{\omega(e)}$, internal

4 Otherwise, the usual squared dependence (31) will not be obtained. 
one $\varepsilon_{0} \propto \sqrt{\omega}$ and elastic one $e$ are connected by the following relation:

$$
\varepsilon^{2}=\varepsilon_{0}^{2}\left(1+e^{2}\right), \quad e \ll 1
$$

The key point is that Eq.(29) supposes the additivity rule holds not for quantities $\varepsilon, \varepsilon_{0}$, e themselves, but for their squares. The physical reason for such a situation is that the system under consideration is random in character and described by a symmetrical distribution function. Therefore, all odd-power moments vanish identically and making use of the additivity rule (29) for variances follows.

It is worthwhile to note the seeming contradiction between above relations for the localization parameter $\omega$ and the Deam-Edwards results [11]. Obvious reason consists in that the formers are obtained within framework of the meanfield theory, whereas the latters suppose fluctuation effects. According to [11] the cross-linking process is not sensible to strain $\varepsilon$ and corresponding localization parameter $\omega$ is proportional to the cross-link number $N$ but not to the difference $N-N_{c}$, as in Eqs.(25), (26). In our opinion, this is caused by nonself-consistency of the approach [11] in sense that the stress field $\sigma$ is switched off. On the other hand, making use of the statistical scheme [11] arrives at the strain-dependence for the localization parameter $\omega$ due to appearance of the polymer network entanglement, whereas within the above phenomenological approach this dependence has to be postulated.

A contribution to the elastic energy caused by the external strain is determined by equality

$$
\Delta E(e) \equiv\left|E(\varepsilon(e))-E\left(\varepsilon_{0}\right)\right|
$$

With use of Eqs. (22), (27), (29) and expansion $\ln (1+x) \approx x-x^{2} / 2+x^{3} / 3, x \ll$ 1 the expression

$$
\Delta E(e) \approx N_{0} \frac{T \epsilon^{3}}{2} e^{2}
$$

is easily obtained where only the first non-zero term is kept. Comparing this relation with the usual expression for the elastic energy [1]

$$
\Delta E(e) \equiv V \frac{G}{2} e^{2}
$$

where $V$ is volume, we arrive at the final expression for the shear modulus of 
the gel state of the polymer network:

$$
G=\frac{T \epsilon^{3}}{\Omega}, \quad \epsilon \equiv \frac{N_{0}-N_{c}}{N_{c}} \ll 1, \quad \Omega \equiv \frac{V}{N_{0}}
$$

The notable peculiarity of this result consists in that, in accordance with previous result (11), the shear modulus is proportional to the third power of the distance $\epsilon$. It is worthwhile to note that an expression of this kind can be obtained only within framework of the synergetic approach used, but not on the basis of the phase transition theory by Landau.

Finally, comparing Eqs. (11) and (33), we obtain the relation between the micro- and macroscopic parameters of the gel under consideration in the proximity of the critical point $(\epsilon \ll 1)$ :

$$
G=g \frac{\xi}{\mathcal{N}} \frac{T}{\Omega}, \quad g \equiv \frac{3}{2} f^{-1} \frac{(f-2)^{2}}{(f-1)^{4}} .
$$

\section{References}

[1] L. D. Landau, E. M. Lifshitz, Elasticity Theory (Nauka, Moscow, 1987).

[2] A. Havranek, M. Marvan, Ferroelectrics 176, 25 (1996).

[3] A. I. Olemskoi, Theory of Structure Transformations in Non-equilibrium Condensed Matter (NOVA Science, N.-Y., 1999).

[4] V. G. Bar'yakhtar, A. I. Olemskoi, Sov. Phys. Solid State 33, 2705 (1991).

[5] P. J. Flory, J.Am.Chem.Soc. 63, 3083 (1941).

[6] P. J. Flory, Principles of Polymer Chemistry (Cornell University Press, 1953).

[7] P. J. Flory, Proc.Roy.Soc.London A351, 351 (1976).

[8] P. M. Goldbart, H. E. Castillo, A. Zippelius, Adv. Phys. 45, 393 (1996).

[9] S. Panyukov, Y. Rabin, Phys. Rep. 269, 1 (1996).

[10] S. F. Edwards, H. Takano, E. M. Terentjev, cond-mat/0007270.

[11] R. T. Deam, S. F. Edwards, Phil.Trans.R.Soc. A280, 317 (1976).

[12] S. F. Edwards, P. W. Anderson, J.Phys. F5, 965 (1975).

[13] W. H. Stockmayer, J.Chem.Phys. 11, 45 (1943).

[14] G. R. Dobson, M. Gordon, J.Chem.Phys. 41, 2389 (1964).

[15] G. R. Dobson, M. Gordon, J.Chem.Phys. 42, 705 (1965). 
[16] K. Dušek, Adv.Polym.Sci. 78, 1 (1986).

[17] G. Haken, Synergetics (Springer-Verlag, Berlin, Heidelberg, N.Y., 1983). 